\title{
Characterization of Ultrasound Fields Using a Potential Optical Flow Based Synthetic Schlieren Tomography
}

\author{
Eero Koponen $^{1}$, Jarkko Leskinen ${ }^{1}$, Tanja Tarvainen ${ }^{1,2}$, and Aki Pulkkinen ${ }^{1}$ \\ ${ }^{1}$ Department of Applied Physics, University of Eastern Finland, P.O. Box 1627, Kuopio, 70211, Finland. \\ ${ }^{2}$ Department of Computer Science, University College London, Gower Street, London, WCIE 6BT, UK. \\ eero.koponen@uef.fi, jarkko.leskinen@uef.fi,tanja.tarvainen@uef.fi, aki.pulkkinen@uef.fi
}

\begin{abstract}
Synthetic schlieren tomography is an optical imaging method for characterization of ultrasound fields based on observing bending of light due to acousto-optic effect. In this work, potential optical flow based pressure estimation method is introduced. (C) 2019 The Author(s)
\end{abstract}

OCIS codes: $170.7170,170.3010$.

\section{Introduction}

Ultrasound is a fundamental part of medical diagnostics and also has therapy applications. To guarantee consistent quality of a treatment, the ultrasound devices need to be calibrated. This requires measurement of the ultrasound field that is commonly done using time-consuming and cumbersome hydrophone measurements. Synthetic schlieren $[1,2]$ tomography (SST) is a potential alternative to both visualize and measure ultrasound fields. In SST, ultrasound waves cause changes in the propagation of light due to acousto-optic effect which manifests as apparent optical distortions in an imaged target. The difference of the tomographically captured optically distorted images and a reference image carry information of the pressure field that can be estimated using methods of computational inverse problems.

In this work, advancements to previously introduced SST [1] approach are suggested. A potential optical flow based pressure estimation method using Horn-Shunck [3] type approach combined with Helmholtz equation based regularization is described [4]. The accuracy of the method is validated and compared to previous estimation methods via simulations.

\section{Potential optical flow}

The measured optically distorted image $I^{\delta}$ and reference image $I$ can be related according to a brightness constancy

$$
I^{\delta}(x, y)=I(x+u(x, y), y+v(x, y)),
$$

where $(u, v)$ are absolute displacements of the light ray from a pixel at $(x, y)$. Assuming a thin refractive index field, the displacements can be approximated as

$$
(u(x, y), v(x, y)) \approx D\left(\phi_{x}(x, y), \phi_{y}(x, y)\right)=\frac{D}{n_{0}}\left(\frac{\partial n}{\partial p}\right) \int_{Z} \nabla p(x, y, z) \mathrm{d} z,
$$

where $D$ is the distance between the pressure field $p$ and the imaged target, $n_{0}$ is the ambient refractive index, $\phi_{x}$ and $\phi_{y}$ are the paraxial deflection angles proportional to the projection of the gradient of pressure field, $(\partial n / \partial p)$ is an adiabatic piezo-optic factor relating the change of refractive index to the change in pressure, and $Z$ is the width of the refractive index field. The displacements fields $(u, v)$ can be expressed in terms of a potential function $\psi$ that is proportional to the projection of the pressure field as

$$
(u(x, y), v(x, y))=\nabla \psi(x, y), \quad \psi(x, y)=\kappa \int_{Z} p(x, y, z) \mathrm{d} z,
$$

where $\kappa=\left(D / n_{0}\right)(\partial n / \partial p)$. Using the potential function $\psi$, the brightness constancy can be approximated using a first order Taylor series

$$
I^{\delta}(x, y) \approx I(x, y)+\nabla I(x, y) \cdot \nabla \psi(x, y) .
$$



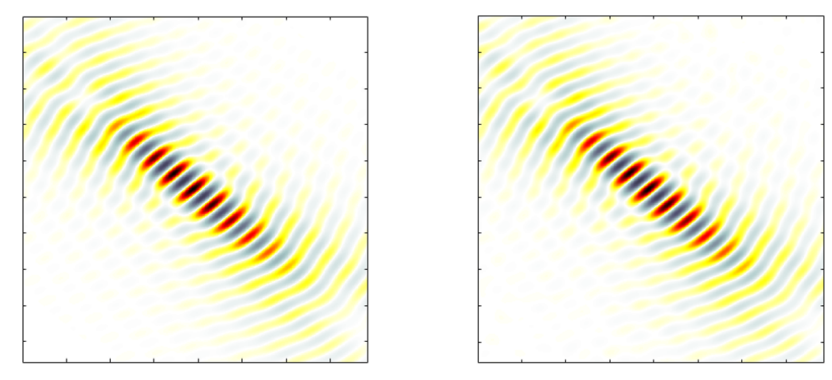

Fig. 1. A simulated obliquely propagating ultrasound wave (left) and potential optical flow based pressure estimation (right) in a $24.40 \times 20.06 \mathrm{~mm}$ region of interest.

Given then measurements $I^{\delta}$ and $I$, the potential function $\psi$ can be estimated using Horn-Shunck type potential optical flow approach. The estimation problem can be expressed as

$$
\hat{\psi}=\underset{\psi}{\arg \min } \int_{A}\left(I^{\delta}-I-\nabla I \cdot \nabla \psi\right) \mathrm{d} x \mathrm{~d} y+\alpha \int_{A} \mathscr{L}\{\psi\}^{2} \mathrm{~d} x \mathrm{~d} y,
$$

where $A$ is the span of the images, $\alpha$ is a regularization parameter, and $\mathscr{L}$ is a regularization operator. For quantities related to acoustic fields, it is justified to define a regularization operator promoting solutions with wave-like features. A Helmholtz equation based regularization operator is

$$
\mathscr{L}\{\psi\}(x, y)=\left(\nabla^{2}+k^{2}\right) \psi(x, y),
$$

where $k$ is the wavenumber of the pressure wave.

\section{Tomographic acoustic pressure estimations}

The estimation problem (5) is solved for each imaged angle $\theta$ over a span of $180^{\circ}$ at $1^{\circ}$ intervals. Hence the estimated projections $\psi$ can be related to pressure $p$ using Radon transform as

$$
\psi\left(x^{\prime}, y, \theta\right)=\kappa \mathscr{R}_{x z}\{p(x, y, z)\}\left(x^{\prime}, \theta\right),
$$

where $\mathscr{R}_{x z}\{\cdot\}\left(x^{\prime}, \theta\right)$ is the Radon transform in a $x z$-plane and $x^{\prime}$ is the rotational axis perpendicular to the direction of the projection. Three-dimensional pressure field is obtained by applying an inverse Radon transform $\mathscr{R}_{x z}^{-1}\{\cdot\}(x, z)$ on each of the two-dimensional sinograms $\psi\left(x^{\prime}, \theta\right)$ in the $y$-direction as

$$
p(x, y, z)=\frac{1}{\kappa} \mathscr{R}_{x z}^{-1}\left\{\psi\left(x^{\prime}, y, \theta\right)\right\}(x, z) .
$$

\section{Results and summary}

The accuracy of the pressure field estimation was validated numerically via simulations for medically relevant ultrasound fields. The results demonstrate that overall, the potential optical flow based approach performs better in feasibility and accuracy in estimating ultrasound fields than traditional optical flow based methods. Fig. 1 shows an example of the estimated pressure field in the case of obliquely propagating ultrasound wave.

\section{Acknowledgements}

This work has been supported by the Academy of Finland (projects 286247, 314411 and 312342 Centre of Excellence in Inverse Modelling and Imaging) and Jane and Aatos Erkko Foundation.

\section{References}

1. A. Pulkkinen, J. J. Leskinen, and A. Tiihonen, "Ultrasound field characterization using synthetic schlieren tomography," J. Acoust. Soc. Am. 141 (6), 4600-4609 (2017)

2. I. Butterworth and A. Shaw, "Realtime scousto-optical QA methods for high intensity fields," in Proceedings of 39th Annual Symposium of Ultrasonic Industry Association, (IEEE, New York, 2010), pp. 1-5.

3. B. K. Horn and B. G. Schunck, "Determining optical flow," Artif. Intell. 17 (1-3), 185-203 (1981).

4. E. Koponen, J. J. Leskinen, T. Tarvainen, and A. Pulkkinen, "Acoustic pressure field estimation methods for synthetic schlieren tomography," J. Acoust. Soc. Am. Submitted (November 2018). 